Michael Bergermann, M.D.,

Paul J. Donald, M.D., F.R.C.S.(C),

and Daniel F. àWengen, M.D.

\title{
Management of Inflammatory Complications of Skull Base Surgery
}

\begin{abstract}
Because the contents of the intracranial cavity are exposed to the upper airway, inflammatory complications are the most frequent in skull base surgery. If dura has to be resected and reconstructed with a graft, the risk of inflammatory complications increases. An evaluation of 93 patients with skull base tumors who were operated on at the University of California, Davis Medical Center between July 1982 and March 1992 and had resection and reconstruction of dura is presented. Inflammatory complications developed in $33(35 \%)$ patients. Local wound infection, cerebrospinal fluid leak, and meningitis are by far the most frequent complications. Patients with middle and combined fossae approaches have the highest incidence. Cause, treatment, and prophylaxis of inflammatory complications are discussed (Skull Base Surgery, 3(1):7-10, 1993)
\end{abstract}

The intact skull base separates the neurocranium from the contaminated upper airway. At the beginning of this century, the skull base was a feared boundary in tumor surgery that was not transgressed. At that time, the risk for disastrous infectious complications was very high in patients with communications between these two spaces, which dissuaded contemporary surgeons from operating in this area.

In the 1960s Ketcham et al ${ }^{1}$ showed that patients with tumors adjacent or penetrating the anterior skull base could be cured by radical surgery when they included resection of the skull base. They also showed that the risk for life threatening complications was tolerable in comparison to the hopeless prognosis of the untreated tumor. In 1966 , they published an overall complication rate of about $75 \%$ in anterior fossa resections. ${ }^{2}$ They included all kinds of complications. Their mortality rate was about $7 \% .2$ In 1985 they published a decreased mortality rate of about $3 \%$.

Since that time, more and more lesions of the skull base have become resectable due to improved techniques, surgical skill, and equipment. Slowly, previously established arbitrary criteria of unresectability have been modified as these factors have evolved. Improvements of reconstructive procedures make more extensive resections possible by establishing a stable separation between the upper aerodigestive tract and the intracranial space. $4-6$ Early and delayed inflammatory complications are still the major cause for postoperative mortality and morbidity and for prolongation of patient stay in the hospital.

Prevention, early recognition, and appropriate treatment of these complications are the mainstay of successful management.

\section{MATERIALS AND METHODS}

We have reviewed the charts of all patients who have undergone extended resection of skull base tumors of the anterior, middle, and posterior fossae at the Department of Otolaryngology - Head and Neck Surgery at the University of California, Davis, between July 1982 and March

Skull Base Surgery, Volume 3, Number 1, January 1993 Department of Otolaryngology-Head and Neck Surgery, University of California, Davis, Davis, California Presented at the First International Skull Base Congress in Hannover on June 18, 1992 Reprint requests: Dr. Donald, Department of Otolaryngology, 2500 Stockton Boulevard, Sacramento, California 95817 Copyright (C) 1993 by Thieme Medical Publishers, Inc., 381 Park Avenue South, New York, NY 10016. All rights reserved. 
1992. Only cases in which the dura was opened, resected, and subsequently reconstructed were included. The cases excluded from the study were simple pituitary gland resections, translabyrinthine acoustic neuroma removal, and cases in which the bony skull base was resected with or without dural exposure but without dural resection.

There were 93 patients ( 54 male, 39 female) included in the study who fulfilled the previously mentioned criteria. The patients' ages were 16 to 82 years. Four patients who fulfilled the requirements but died in the early postoperative period were excluded (two deaths due to brain infarction, one related to cardiac arrest, one of unknown reason). The mortality rate was $4.3 \%$. Fifty patients had malignant tumors.

\section{RESULTS AND DISCUSSION}

Table 1 shows the tumor locations. The histologic diagnosis was squamous cell carcinoma in 23 patients, schwannoma in 21 patients, meningioma in eight patients, adenoid cystic carcinoma in six patients, adenoma in six patients, and adenocarcinoma in four patients. The other tumors had been diagnosed as basal cell carcinoma, esthesioneuroblastoma, fibrosarcoma, malignant melanoma, hemangiopericytoma, inverted papilloma, angiofibroma, glomus tumor, craniopharyngioma, chordoma, chondrosarcoma, fibrous dysplasia, glomus tumor, and metastasis of a renal cell carcinoma. Of these, $43 \%$ were recurrent tumors that had been treated before by surgery, radiation, chemotherapy, or a combination of these.

We were looking for the following inflammatory complications: brain or epidural abscess, chronic aspiration, foreign body reaction, hydrocephalus, keratitis, meningitis, osteomyelitis, pneumocephalus, tissue or flap necrosis, and wound infection (in alphabetical order). Cerebrospinal fluid (CSF) leak, a noninflammatory complication, was included in our study, because it can cause major inflammatory complications. The patients were divided into a group with early onset and a group with delayed onset complications. All complications that appeared later than 14 days postoperatively were defined as "delayed onset." All complications could be allocated to one of these groups. The delayed onset complications often developed after several months.

Table 1. Tumor Location

\begin{tabular}{lcr}
\hline & No. patients & $\%$ \\
\hline Anterior fossa & 21 & 23 \\
Middle fossa & 27 & 29 \\
Posterior fossa & 18 & 19 \\
Anterior/middle fossa & 5 & 5 \\
Middle/posterior fossa & 6 & 7 \\
Clivus and sella & 12 & 13 \\
Temporal bone & 4 & 4 \\
Total & 93 & \\
\hline
\end{tabular}

Tables 2 and 3 show the frequency of inflammatory complications. Most of the patients developing complications initially presented with recurrent disease. The approaches to the middle fossa and those involving multiple fossae had the highest rates of complications. ${ }^{7}$ This seems to be related to the wide exposure necessary to gain access to the tumor-bearing area, the difficulties in archieving watertight dural closure, and a lack of well-vascularized local soft tissue for reconstruction.

The craniofacial approach to the anterior fossa was the most reliable procedure, with a low incidence of complications (Table 4). All craniofacial resections included a frontal craniotomy. The frontal sinus mucosa was removed meticulously and the sinus was cranialized bilaterally. The frontal duct mucosa was inverted and the duct was plugged with a fascia or fat graft. A spinal drain was used in all but one patients for 3 to 5 days. Dura was resected in all cases and reconstructed with autologous fascia or a cadaver graft. An anteriorly or laterally based pericranial flap was routinely used to separate the upper airway from the neurocranium. This flap was covered by a split-thickness skin graft from below. Osteomyelitis of the bone flap developed in three patients $(14 \%)$. These patients had all prior surgery and two of them had received radiation treatment. One patient developed a long-standing pneumocephalus. He did not get either a tracheotomy or a spinal drain during the operation. In addition, dead space between the remnants of the floor of the anterior fossa and the pericranial flap had not been obliterated with a free fat graft. About 2 years after the tumor resection the patient still has an extensive pneumocephalus without any clinical symptoms. Presently, all patients have a tracheotomy with a cuffed tracheotomy tube for 7 days and a spinal drain for 3 to 5 days postoperatively.

Table 2. Patients with Early Onset Complications

\begin{tabular}{lrrc}
\hline & No. & $\%$ & Previous Treatment \\
\hline Anterior fossa & 1 & 5 & 0 \\
Middle fossa & 13 & 48 & $9(69 \%)$ \\
Posterior fossa & 5 & 28 & 0 \\
Anterior/middle fossa & 2 & 40 & $2(100 \%)$ \\
Middle/posterior fossa & 3 & 50 & $3(100 \%)$ \\
Clivus and sella & 1 & 9 & 0 \\
Temporal bone & 1 & 20 & 0 \\
\hline
\end{tabular}

Table 3. Patients with Delayed Onset Complications

\begin{tabular}{lccc}
\hline & No. & $\%$ & Previous Treatment \\
\hline Anterior fossa & 4 & 19 & $4(100 \%)$ \\
Middle fossa & 4 & 15 & $3(75 \%)$ \\
Posterior fossa & 2 & 12 & 0 \\
Anterior/middle fossa & 1 & 20 & $1(100 \%)$ \\
Middle/posterior fossa & 0 & & 0 \\
Clivus and sella & 0 & & 0 \\
Temporal bone & 0 & & 0 \\
\hline
\end{tabular}


Table 4. Anterior Fossa (Patients with Complications)

\begin{tabular}{lll}
\hline & Early & Delayed \\
\hline Cerebrospinal fluid leak & 0 & $1(5 \%)$ \\
Meningitis & 0 & 0 \\
Epidural abscess & 0 & $1(5 \%)$ \\
Wound infection & 0 & $1(5 \%)$ \\
Tissue necrosis & 0 & 0 \\
Pneumocephalus & $1(5 \%)$ & $1(5 \%)$ \\
Osteomyelitis & 0 & $3(14 \%)$ \\
Hydrocephalus & 0 & 0 \\
Chronic aspiration & 0 & 0 \\
Keratitis & 0 & 0 \\
\hline
\end{tabular}

In our series the risk for inflammatory complications was increased in patients with middle cranial fossa resections (Table 5). One reason for that is the difficulty in achieving a watertight dural closure. Such a closure is often impossible, especially around the cavernous sinus. Even by lowering the CSF pressure with a spinal drain, the risk for CSF leak is high in these patients. In addition, after extended soft tissue resection in the infratemporal fossa, there is often not enough well-vascularized soft tissue available to obliterate all dead spaces. The lack of soft tissue makes this area less resistent to wound breakdown followed by CSF leak and meningitis. The use of revascularized free flaps offers an excellent solution to this dilemma. All four patients reconstructed with free flaps had no major postoperative or delayed complications.

The eustachian tube was completely resected after exposing the middle ear space or carefully plugged with a muscle graft in all patients. Together with the placement of tympanostomy tubes, these procedures prevented otits media in all patients

An unusual complication was the development of a foreign body granuloma in the sphenoid sinus that necessitated a second operation because it looked like recurrent tumor. In this patient the sphenoid sinus had been packed with Oxycel for hemostasis and the material caused a foreign body reaction.

All patients received perioperative intravenous gentamicin and clindamycin.

Posterior fossa approaches had a low incidence of

Table 5. Middle Fossa (Patients with Complications)

\begin{tabular}{lll}
\hline & Early & Delayed \\
\hline Cerebrospinal fluid leak & $4(15 \%)$ & $1(4 \%)$ \\
Meningitis & $4(15 \%)$ & $2(7 \%)$ \\
Brain abscess & 0 & 0 \\
Wound infection & $8(33 \%)$ & 0 \\
Tissue necrosis & $2(7 \%)$ & 0 \\
Pneumocephalus & $1(4 \%)$ & 0 \\
Osteomyelitis & 0 & $2(7 \%)$ \\
Hydrocephalus & $1(4 \%)$ & $1(4 \%)$ \\
Mucocele & 0 & 0 \\
Chronic aspiration & 0 & 0 \\
\hline
\end{tabular}

Table 6. Posterior Fossa (Patients with Complications)

\begin{tabular}{lll}
\hline & Early & Delayed \\
\hline Cerebrospinal fluid leak & $1(6 \%)$ & 0 \\
Meningitis & $1(6 \%)$ & $1(6 \%)$ \\
Brain abscess & 0 & 0 \\
Wound infection & 0 & 0 \\
Tissue necrosis & 0 & 0 \\
Pneumocephalus & 0 & 0 \\
Osteomyelitis & 0 & 0 \\
Hydrocephalus & $1(6 \%)$ & 0 \\
Chronic aspiration & 0 & $1(6 \%)$ \\
Keratits & $2(11 \%)$ & 0 \\
\hline
\end{tabular}

inflammatory complications (Table 6). Most of the patients had slow-growing benign tumors without previous treatment. In these patients the brain cavity can be sealed off from the aircell system of the temporal bone without difficulties. Free fat and fascia grafts were used to seal dura defects. The transtemporal bone and the transphenoidal approaches were almost free of inflammatory complications.

The group of patients with combined procedures involving more than one fossa had an inflammatory complication rate of $40 \%$ (Tables 7 and 8 ). With one exception these approaches had been used to resect extensive recurrent lesions. These lesions had usually been treated before with multiple modalities. These were the most timeconsuming procedures, sometimes done in two stages. There was always a lack of local well-vascularized soft tissue for reconstruction. Although this group of $11 \mathrm{pa}-$ tients is too small for a statistical analysis, the use of free flaps seemed to decrease the complication rate.

\section{CONCLUSIONS}

Of 93 patients who had undergone resection of skull base tumors with opening and resection of dura, 35\% developed inflammatory complications. The appearance of complications is related to tumor location and extension. ${ }^{7}$ Most of these complications could be handled by conservative treatment, including prolonged use of anti-

Table 7. Anterior/Middle Fossa (Patients with Complications)

\begin{tabular}{lll}
\hline & Early & Delayed \\
\hline Cerebrospinal fluid leak & 0 & 0 \\
Meningitis & $1(20 \%)$ & 0 \\
Epidural abscess & 0 & 0 \\
Wound infection & 0 & $1(20 \%)$ \\
Tissue necrosis & 0 & 0 \\
Osteomyelitis & 0 & $1(20 \%)$ \\
Hydrocephalus & 0 & 0 \\
Keratitis & $1(20 \%)$ & 0 \\
\hline
\end{tabular}


Table 8. Middle/Posterior Fossa

(Patients with Complications)

\begin{tabular}{llc}
\hline & Early & Delayed \\
\hline Cerebrospinal fluid leak & $1(17 \%)$ & 0 \\
Meningitis & $1(17 \%)$ & 0 \\
Epidural abscess & $1(17 \%)$ & 0 \\
Wound infection & $1(17 \%)$ & 0 \\
Tissue necrosis & $1(17 \%)$ & 0 \\
Osteomyelitis & 0 & 0 \\
Hydrocephalus & $1(17 \%)$ & 0 \\
Keratitis & 0 & 0 \\
\hline
\end{tabular}

biotics, spinal drainage, and wound debridement. Two patients required surgical treatment of long-standing CSF leaks.

For 3 years, we have implanted gold weights into the paralyzed upper lid to prevent keratitis. No case of keratitis was seen after we started to use this technique. 8,9

Chronic aspiration is treated with Gelfoam or Teflon injection into the paralyzed vocal cord. One patient who also had a pharyngeal resection needed a laryngectomy to prevent aspiration. Osteomyelitis was seen in patients who had been preoperatively treated with multiple modalities. These patients needed long-standing and time-consuming local wound care or further surgical procedures. ${ }^{10}$ The perioperative use of high-dose antibiotics (intravenous clindamycin and Gentamycin) was routine in all cases with opening of the upper aerodigestive tract. ${ }^{11}$

Only one patient died of inflammatory complications. He developed recurrent CSF leaks despite several conservative and surgical attempts to close the fistula. He died of suppurative meningitis 14 months after resection of a brain-invading squamous cell carcinoma.

The pedicled or free transfer of nonirradiated wellvascularized tissue to seal off the neurocranium from the contaminated upper airway and to fill any dead space are the keystones in the prevention of inflammatory complica- tions in skull base operations with dural resection. The pericranial flap is the workhorse in anterior fossa resections. ${ }^{12}$ The temporalis muscle flap serves the same function in middle and posterior fossa resections as long as the blood supply to the muscle is preserved. In combined approaches, especially including a neck dissection with removal of the external carotid artery, the use of bulky revascularized free flaps reduces the risk for inflammatory complications.

\section{REFERENCES}

1. Ketcham AS, Wilkins RH, Van Buren JM, Smith RR: A combined intracranial approach to the paranasal sinuses. Am J Surg 106: 698-703, 1963

2. Ketcham AS, Hoye RC, Van Buren JM, Johnson RH, Smith RR: Complications of intracranial resection for tumors of the paranasal sinuses. Am J Surg 112:591-596, 1966

3. Ketcham AS, Van Buren JM: Tumors of the paranasal sinuses. Am J Surg 150:406-413, 1985

4. Jones NF, Schramm VL, Sekhar LN: Reconstruction of the cranial base following tumor resection. Br J Plast Surg 40:155-162, 1987

5. Schuller DE, Goodman JH, Miller CA: Reconstruction of the skull base. Laryngoscope 94:1359-1364, 1984

6. Jackson CG, Glasscock ME, McKennan CF, Levine SC, Hays JW, Smith HP: The surgical treatment of skull base tumors with intracranial extension. Head Neck Surg 96:175-185, 1987

7. Wornom IL, Neifeld JP, Mehrhof AI, Young HF, Chincilli VN: Closure of craniofacial defects after cancer resection. Am J Surg 162:408-411, 1991

8. May M: Gold weight and wire spring implants are alternative to tarsorrhaphy. Arch Otolaryngol Head Neck Surg 113:656-660, 1987

9. Keen M, Vitale V, Post K: Immediate reversible rehabilitation of the paralyzed eyelid. Skull Base Surg 2:28-37, 1992

10. Komisar A, Silver C, Kalnicki S: Osteoradionecrosis of the maxilla and skull base. Laryngoscope 95:24-28, 1985

11. Carrau RL, Synderman C, Janecka IP, Sekhar L, Sen C, D'Amico F: Antibiotic prophylaxis in cranial base surgery. Head Neck 13: 311-317, 1991

12. Price JC, Loury M, Carson B, Johns ME: The pericranial flap for reconstruction of anterior skull base defects. Laryngoscope 98: 1159-1164, 1988 